\title{
De l'éthique environnementale au principe responsabilité et retour
}

Hicham-Stéphane Afeissa

\section{(2) OpenEdition \\ Journals}

Édition électronique

URL : http://journals.openedition.org/ere/2084

DOI : 10.4000/ere.2084

ISSN : 2561-2271

Éditeur

Centr'ERE

Référence électronique

Hicham-Stéphane Afeissa, " De l'éthique environnementale au principe responsabilité et retour », Éducation relative à l'environnement [En ligne], Volume 8 | 2009, mis en ligne le 20 décembre 2009, consulté le 21 février 2020. URL : http://journals.openedition.org/ere/2084; DOI : 10.4000/ere.2084 


\title{
De l'éthique environnementale au principe responsabilité et retour
}

\author{
Hicham-Stéphane Afeissa
}

1 Sans doute serait-il erroné de dire que l'éthique environnementale anglo-américaine a encore mauvaise presse en France de nos jours. Le temps des imprécations tonitruantes et des mises en garde solennelles est heureusement révolu. Mais force est de constater que l'accueil assez réservé qui lui a été fait, en un peu plus d'une décennie, non seulement en France, mais également dans quelques autres pays du continent européen, ne lui a pas encore permis de se faire bien connaître de celles et ceux qui sont sensibles aux questions d'environnement. Tout se passe comme si un océan de pensée retenait à domicile, dans les Amériques, les problématiques élaborées par l'éthique environnementale, autour desquelles aucun programme de recherche bien défini ne s'est à l'heure actuelle développé en Europe, où d'autres problématiques sont privilégiées.

2 Les deux types d'approches des problèmes environnementaux continuent ainsi leur petit bonhomme de chemin l'un à côté de l'autre, chacun n'ayant d'égards que pour soimême, sans véritablement réussir à se croiser et à tirer un bénéfice de leurs différences mêmes. Le paradoxe veut que des œuvres aussi importantes et novatrices que celles de Hans Jonas, de Ulrich Beck et de Bruno Latour, d'une part, et de Holmes Rolston III, de J. Baird Callicott et de Bryan G. Norton, de l'autre, qui sont fort éloignées au point de s'ignorer les unes les autres au sein de leur communauté philosophique d'origine, semblent ne plus rien avoir à se dire, passé la ligne des monts, le cours d'un fleuve ou l'espace d'une mer, comme si leur objet de réflexion n'était pas au fond le même.

3 Nous souhaiterions dans ce qui suit contribuer à délimiter le périmètre d'un espace commun d'interlocution, en nous efforçant de définir les conditions d'un dialogue que nous voudrions fructueux entre ces différents modes d'approche des problèmes environnementaux. Pareille entreprise implique en tout premier lieu de comprendre exactement en quoi consiste la spécificité de chacun de ces deux courants de pensée que - par commodité et quitte à rectifier cette hypothèse de travail subséquemment nous supposerons suffisamment homogène et unifiée autour de quelques grandes 
problématiques pour permettre de les distinguer l'un de l'autre. Cette tâche effectuée, nous tenterons alors de mettre au jour de possibles points de jonction, non pas toutefois en accompagnant, pour ainsi dire, un mouvement de convergence qui n'attendrait que d'être révélé pour s'effectuer pleinement, mais bien en le forçant au moyen d'une analyse critique dénonçant, d'une part, le défaut de radicalité constitutif de la méthode de problématisation prédominante dans le continent européen, et, d'autre part, le risque de blocage idéologique auquel s'expose l'éthique environnementale en mettant au centre de toutes ses élaborations le concept de valeur intrinsèque des entités du monde naturel. À terme, nous espérons que les insuffisances de l'une et l'autre approche pourront, d'une certaine manière, s'annuler au regard d'une théorie écologique d'inspiration pragmatique, par l'esquisse de laquelle nous refermerons notre enquête.

\section{Risques environnementaux et responsabilités éthiques : le modèle de l'action}

4 Il serait intéressant de chercher à montrer en détail de quelle manière la réflexion écologique en Europe, au cours des trois dernières décennies, a pris le relais dans une large mesure de la réflexion angoissée qu'a pu susciter à un certain moment la création et l'usage des technologies modernes.

5 Dans la perspective de la reconstitution d'une telle généalogie, il conviendrait sans doute de réserver une place importante à l'élucidation par Martin Heidegger (1980) de l'essence de la technique en termes d' " arraisonnement » du monde, de même qu'à la méditation de Karl Jaspers (1963) sur les dangers que fait courir à «l'existence en général » la bombe atomique, ainsi qu'au cri d'alarme lancé par Günther Anders (2006) annonçant que le temps de la fin du monde est venu en cette époque où l'arme nucléaire a fait de la destruction de la planète une possibilité permanente. Mais ce qu'il importerait particulièrement de montrer, nous semble-t-il, est que pareille filiation n'est pas seulement ni fondamentalement de nature rhétorique - au sens où une prophétie de malheur viendrait alors se substituer à une autre, dans la plus pure tradition des discours apocalyptiques et catastrophistes -, mais qu'elle est d'abord et avant tout de nature substantielle, en ce sens où c'est bien le même objet de réflexion qui est examiné.

6 A-t-on suffisamment remarqué que Hans Jonas lui-même, l'auteur de la première grande éthique de la nature du XXe siècle (éthique dont nous aurons bientôt à évaluer la portée écologique), indique expressément l'existence d'une telle filiation à l'origine de sa réflexion sur les problèmes environnementaux, non seulement dans les textes où il évoque furtivement «l'ombre de la Bombe » (Jonas, 1996, p. 128), mais encore dans ceux, particulièrement précieux, où il livre une sorte d'autobiographie intellectuelle, et dans lesquels « l'impact que toute la technique de l'humanité d'aujourd'hui exerce sur l'environnement naturel» est mentionné au terme d'un long développement qui s'ouvre par l'évocation d'Hiroshima et de «la course aux armements atomiques» (Jonas, 1998, p. 49-52)?

7 L'héritage que la critique philosophique de la technique marquée du signe de l'angoisse a légué à la pensée de Hans Jonas - et au-delà de cette dernière, ainsi que nous allons tenter de le montrer, au courant de réflexion écologique de style euro-continental - 
réside pour l'essentiel, à nos yeux, dans la primauté accordée aux problèmes que pose l'usage des techniques, et dans la nécessité proclamée de redéfinir des règles de responsabilité qui soient à la mesure des nouveautés introduites par le développement technologique.

Plus précisément, il nous semble que la grande originalité de Hans Jonas est d'avoir su déplacer le centre de gravité de la réflexion en tirant parti des analyses menées par Hannah Arendt (1983), et notamment de la distinction capitale que propose celle-ci entre le travail de notre corps, l'œuvre de nos mains et l'action proprement dite, qui pourrait bien avoir joué dans la genèse de la pensée de Jonas un rôle plus important que la distinction aristotélicienne, généralement invoquée par les interprètes, entre poiésis (acte technique au service d'une fin) et praxis (acte moral qui a sa fin en luimême).

9 Comme le fait justement remarquer Hans Jonas, ce n'est pas l'usage militaire de la fission nucléaire qui est proprement inquiétant, puisque à bien y regarder «l'usage pacifique quotidien que nous faisons de notre pouvoir [sur la nature] et sur lequel repose toute notre existence civilisée avec son confort (conduire sa voiture, prendre l'avion, etc.), avec l'abondance extraordinaire de biens dont nous disposons » (Jonas, 1991, p.11) l'est au moins tout autant, si ce n'est plus; ce n'est pas non plus la dimension planétaire et le long terme des effets cumulatifs qui doit préoccuper. Les accidents technologiques, industriels, nucléaires, chimiques ou pétroliers, c'est-à-dire les effets secondaires ou les erreurs de conception et de gestion, ne sont que les manifestations spectaculaires d'un bouleversement qui est autrement plus profond, et dont nous commençons à avoir un pressentiment à la considération des effets ambivalents des technologies utilisées à bon escient et pour des fins louables. L'étonnant est que, à une certaine échelle, même la logique du succès finisse par s'invertir et les effets attendus par devenir eux-mêmes pervers :

L'action a lieu dans un contexte où tout emploi à grande échelle d'une capacité engendre, en dépit de l'intention droite des agents, une série d'effets liés étroitement aux effets «bénéfiques » immédiats et intentionnés, série qui aboutit, au terme d'un processus cumulatif, à des conséquences néfastes dépassant parfois de loin le but recherché. (Jonas, 1997, p. 232)

10 Ce que révèlent les effets insidieux et involontaires d'actions motivées et réalisées pour le mieux, c'est, pour le dire cette fois-ci avec Hannah Arendt, que «nous avons commencé à agir à l'intérieur de la nature comme nous agissions à l'intérieur de l'histoire» (Arendt, 1972, p. 80). En effet, nous savons aujourd'hui que nous sommes capables, en libérant certaines forces naturelles, de déclencher de nouveaux processus naturels qui n'auraient jamais existé sans l'intervention directe de l'action humaine (que l'on songe, par exemple, à la technologie nucléaire, mais encore aux manipulations génétiques, aux nanotechnologies, etc.), et qu'en un sens par conséquent nous « faisons la nature », dans la même mesure que nous « faisons l'histoire ».

11 Le point important est de bien comprendre que, en captant la nature dans le monde humain, nous n'avons pas seulement accru notre pouvoir sur la nature, nous ne sommes pas seulement devenus plus agressifs dans notre rapport à la nature, nous avons effacé les frontières entre la nature et la société et introduit dans le règne de la nature l'imprévisibilité constitutive de l'action humaine, laquelle, à la grande différence de tout processus de fabrication, ne laisse pas de produit achevé derrière elle, mais provoque bien plutôt une cascade créatrice (et parfois destructrice) d'événements en chaîne dont l'issue reste indéterminable. "Agir dans la nature, 
transporter l'imprévisibilité humaine dans un domaine où l'on est confronté à des forces élémentaires qu'on ne sera peut-être jamais capable de contrôler sûrement " (Arendt, 1972, p. 85), tel est en son essence, selon Arendt, le danger auquel nous confronte la technologie moderne.

Arendt et Jonas nous paraissent donc, sur ce point, parler d'une seule voix. Le problème que pose le développement technologique tient à la corrélation entre la puissance et la prise de risque, en sorte que désigner la puissance d'agir en tant que telle est au fond plus important que de lui donner un signe (négatif ou positif) en regard des fins au service desquelles elle serait mise. Jonas est peut-être le premier à avoir compris que la crise environnementale à laquelle nous sommes confrontés résulte du fait que la différence conceptuelle entre une action humaine et un événement naturel s'est évanouie, à telle enseigne qu'il n'est plus possible désormais de ne pas chercher à tenir compte des effets indirects imprévisibles de nos actions intentionnelles.

Nous avons atteint un stade où il nous incombe de répondre de cela même dont personne n'a jamais voulu l'avènement (le réchauffement climatique, l'érosion de la biodiversité, etc.) - responsabilité à laquelle Jonas donne force de principe, et que décline à sa manière, de nos jours, le fameux principe de précaution, lequel est en effet tout entier tourné vers une appréhension des effets indirects de nos actions techniques, vers une recherche systématique des effets involontaires, et non pas seulement des bénéfices prévisibles escomptés. Dès lors que la nature est devenue partie prenante de l'histoire entendue comme processus où se joue l'action humaine, la responsabilité doit être redéfinie de telle sorte à ne plus simplement désigner l'imputation à un agent d'une action passée, mais une attribution après coup qui engage l'avenir dans sa globalité, dans la mesure où nous avons à répondre dès à présent des générations futures.

14 Il vaut d'être remarqué que le diagnostic jonassien est étonnamment proche de celui qu'avance Ulrich Beck au sujet de la désormais fameuse «société du risque » (Beck, 2001). En effet, lui aussi estime que nous sommes entrés de plain-pied dans une nouvelle forme de société caractérisée par les implications imprévisibles de nos interventions technologiques dans la nature à l'échelle mondiale. Selon le sociologue munichois, l'explosion de la centrale nucléaire de Tchernobyl, l'épidémie d'encéphalite spongiforme bovine, par exemple, ne sont pas des événements qui se produiraient de manière simplement accidentelle, mais elles sont plutôt des conséquences inhérentes produites non intentionnellement par la modernisation même. Contrairement aux catastrophes naturelles de la société préindustrielle, qui étaient le fruit du " hasard", et aux dangers de la société industrielle - qui étaient, eux, le résultat de décisions sociales et politiques, limités dans le temps et dans l'espace, et qui étaient perceptibles, prévisibles, contrôlables et assurables -, les risques de la société post-industrielle sont fabriqués socialement, non prévus et relativement non contrôlables (que l'on songe, par exemple, aux impondérables de la commercialisation des organismes génétiquement modifiés). Ces nouveaux risques - insiste Ulrich Beck - ne sont pas des effets externes, mais bien des effets internes à la société, au titre de répercussions systémiques de l'industrialisation globale.

15 Le point remarquable de toutes ces analyses tient à nos yeux à ce que Ulrich Beck puisse interpréter la situation inédite de la modernité dans les termes mêmes de Jonas et d'Arendt, à savoir comme le signe d'un effacement de la frontière entre la nature et le domaine du politique. Les risques environnementaux - dit-il - sont «politiquement 
réflexifs ", c'est-à-dire qu'ils expriment la manière dont une société se met elle-même en danger dans la relation qu'elle soutient avec une nature dont les processus sont amenés dans l'artifice humain au point d'être désormais indissociables du domaine des affaires humaines. «On assiste [de nos jours] à la fin de l'opposition entre nature et société ", écrit Ulrich Beck, dans la mesure où « les destructions de la nature, intégrées dans la circulation universelle de la production industrielle, [ont] cess[é] d'être de "simples » destructions de la nature pour devenir partie intégrante de la dynamique sociale, économique et politique» (Beck, 2001 p. 146). La nature est devenue politique, et réciproquement toute politique devra dorénavant être une politique de la nature.

Dans le cadre de la modernité avancée, il est justement devenu impossible de comprendre la société et tous ses systèmes partiels - économie, politique, famille, culture - indépendamment de la nature. Les problèmes d'environnement ne sont pas des problèmes qui se jouent dans les «environs ", ce sont incontestablement dans leur genèse comme dans leur forme - des problèmes sociaux, des problèmes de l'Homme, qui touchent à son histoire, à ses conditions de vie, à son rapport au monde et à la réalité, à son organisation économique, culturelle et politique. (Beck, 2001, p. 148)

C'est clairement dans cette même ligne de réflexion que s'inscrit le dernier auteur que nous citions en introduction, lequel, après la mort de Dieu, proclame la bonne nouvelle de la mort de la nature entendue comme nature unifiée, objective, définissant une réalité socialement transcendante étrangère comme telle à tout processus historique et civilisationnel. Bruno Latour - puisque c'est de lui qu'il s'agit - fait même de cette oraison funèbre la condition préjudicielle d'un nouveau départ de la philosophie politique, qui romprait enfin avec la séparation conceptuelle des pouvoirs qui est aussi bien une séparation idéologique puisque la reconnaissance de la neutralité objective de la nature (et de la science en tant que forme privilégiée de connaissance de la nature) est, selon lui, le stratagème qui a toujours permis à la «nature » d'être mobilisée de façon partisane pour mettre un terme aux débats politiques. De ce point de vue, comme l'écrit Bruno Latour, «il n'y a jamais eu d'autre politique que celle de la nature et d'autre nature que celle de la politique " (Latour, 1999, p. 46), dans la mesure où la nature, en tant que réalité extérieure objective, n'a jamais cessé d'être un objet construit pour répondre à des fins politiques.

Si donc la crise écologique contemporaine peut prétendre d'une manière ou d'une autre adresser au philosophe une convocation à penser, ce ne peut être qu'à la condition que la crise environnementale se laisse interpréter comme une crise d'objectivité, qui interdise une bonne fois pour toutes de revenir à la représentation de deux ensembles distincts - la nature d'un côté et la société de l'autre. L'avenir de l'écologie politique dépend entièrement de la capacité des mouvements écologiques à repousser une " conception de la nature qui rend impraticable leur combat politique " (Latour, 1999, p. 34), ce qui implique de leur part qu'ils apprennent à mobiliser à des fins politiques diverses "natures " dans le cadre d'une nouvelle constitution a-moderne, laquelle commencera par reconnaître l'existence d'entités hybrides (tels le trou d'ozone ou la fonte de la calotte polaire) qui ne sont à proprement parler ni des choses naturelles ni des constructions sociales, mais d'une certaine manière les deux à la fois, puisque leur mode d'existence ressortit simultanément à l'ontologie de l'agir humain et à celle des processus naturels qui échappent à tout contrôle humain.

Une fois cette reconnaissance acquise, il importera, dans la perspective de l'écologie politique que Bruno Latour appelle de ses vœux, de comprendre que le problème posé 
par les objets hybrides ne tient pas au caractère potentiellement dangereux de leurs effets (bien que certains aient effectivement de tels effets), mais plutôt à ce qu'ils ne sont pas encore politiquement représentés. En rendant «invisible, impensable, irreprésentable le travail de médiation qui assemble les hybrides » (Latour, 1991, p. 53), la constitution moderne les a par là même exonéré de tout examen public, de toute évaluation et de toute régulation délibératives. Le défi que doit relever l'écologie politique contemporaine, selon Latour, consiste donc à édifier « un parlement des choses ", c'est-à-dire à donner un siège aux hybrides dans nos assemblées représentatives, dans lesquelles des savants, des écologistes, des urbanistes, des météorologues et des citoyens ordinaires pourront débattre et légiférer au sujet des hybrides en vue de substituer à la folle prolifération actuelle des hybrides une production qui soit réglée et décidée en commun - moyennant quoi, comme l'ont justement souligné récemment les auteurs d'une étude importante sur le principe de précaution, Bruno Latour peut bien être tenu pour "le philosophe qui a le mieux exprimé la situation politique qui a donné naissance » (Ewald et al., 2001, p. 42) au dit principe.

\section{L'anthropocentrisme des valeurs et l'éthique environnementale}

19 Il va de soi que nous n'entendons pas contester que pareille approche puisse être réellement éclairante ni qu'elle puisse permettre d'apporter de précieux éléments à la compréhension des problèmes environnementaux. Mais il nous semble que l'approche qui prévaut aujourd'hui, et que nous avons tenté d'unifier autour de quelques idées clés, en comparaison avec les problématiques qu'élabore l'éthique environnementale, souffre d'un défaut de radicalité, dans la mesure où la nature et les causes profondes de la «crise écologique » à laquelle nous sommes confrontés restent, dans ce cadre de réflexion, insuffisamment interrogées.

En quel sens peut-on parler en effet de « crise » environnementale? Au sens - dira-t-on - d'une perturbation qui défie les possibilités de reconstitution des stocks naturels et de restauration de ses équilibres. Les interventions humaines, parce qu'elles sont de plus en plus massives et de plus en plus concentrées dans le temps, menacent d'interrompre des cycles naturels et de nous conduire à des seuils d'irréversibilité - dangers auxquels il est possible de se soustraire à la condition que les politiques environnementales se fixent expressément pour objectif d'analyser et de critiquer les principes d'action qui portent en eux le risque d'une rupture d'équilibre, puis de prescrire de nouvelles règles de conduite conformes aux leçons de l'écologie et d'autres disciplines scientifiques, sans pour autant conférer à la science un pouvoir de décision en dernière instance.

21 Mais est-ce poser convenablement le problème de la crise de l'environnement que de le faire dans le langage de la compatibilité énergétique, c'est-à-dire sous la forme de cette tenue de livres, à laquelle la crise a effectivement d'abord donné lieu (que l'on songe par exemple aux travaux du Club de Rome, avec ses interminables colonnes de chiffres), comme s'il s'était agi, pour l'Homme occidental, de faire ses comptes avec la nature, sans que jamais soient examinés pour eux-mêmes les principes et les motifs des rapports que nous soutenons avec elle ? La position qui consiste à situer le danger dans l'activité humaine (même redéfinie dans la perspective de la philosophie de Hannah Arendt), et qui par là pense pouvoir résoudre tous les problèmes en modifiant cette activité à la 
lumière de principes rationnels et scientifiques, ne présuppose-t-elle pas que le cadre général de nos relations à la nature, quoique mal délimité, reste pour le fond adéquat?

L'une des convictions que partagent bon nombre d'éthiciens de l'environnement est qu'il est nécessaire d'interroger explicitement les modalités générales du rapport à la nature tel qu'il a été pensé par la tradition philosophique, morale, scientifique et religieuse occidentale, si bien qu'il ne nous soit plus possible de puiser sereinement dans cette tradition les éléments permettant de fournir une solution aux problèmes écologiques auxquels nous sommes confrontés, dans la mesure où cette tradition fait ellemême partie du problème.

Cette mise en cause massive de l'ensemble de la tradition occidentale, considérée de telle façon que les phénomènes de crise paraissent résulter de ce qui lui est le plus propre, est sans doute ce qui définit le mieux l'exacte portée du questionnement en éthique environnementale, et ce qui permet immédiatement de comprendre qu'elle n'est ni une forme d'éthique appliquée au domaine particulier de l'environnement, prenant place à côté de l'éthique appliquée des affaires, de l'éthique appliquée d'entreprise, et autres éthiques d'accompagnement du développement industriel, ni une éthique spécialisée ou sectorielle, à l'instar de la bioéthique qui pourrait se contenter d'appliquer le modèle de l'une des morales normatives disponibles ( $d$ 'inspiration utilitariste, déontologique, etc.) aux problèmes éthiques inédits suscités par le développement technologique, en soulevant la question des normes et des règles qui doivent lui servir de limites.

24 En effet, définie de façon générale, l'éthique environnementale est une entreprise qui se donne pour programme d'examiner systématiquement les valeurs qui règlent le rapport de l'être humain à son environnement naturel, et pour objectif de restreindre sur la base de normes la classe des actions permises à l'égard de la nature. La régulation qui doit être mise en place dans notre rapport à la nature n'est pas d'abord et fondamentalement une régulation de type juridico-politique, mais bien une régulation morale qui détermine les conditions sous lesquelles il est légitime d'étendre la communauté des êtres et des entités à l'endroit desquels les êtres humains doivent se reconnaître des devoirs, de la forme de vie animale la plus fruste à l'ensemble des écosystèmes qui composent notre environnement naturel.

25 Si pareille entreprise exige l'adoption d'une nouvelle attitude éthique, impliquant une refonte des postulats philosophiques, théologiques, etc., à la base de la représentation de l'être humain dans la nature, ainsi qu'un remaniement de la société en ses modes de vie et d'administration économique et politique, alors il ne peut suffire d'appliquer telle ou telle morale aux problèmes environnementaux, mais il convient bien plutôt de s'interroger sur les conditions sous lesquelles une morale comme telle peut être constituée, du type d'obligation qu'elle est censée prescrire, du genre de preuve qu'on est en droit d'attendre de sa part, de l'aide qu'elle est capable d'apporter dans le processus de décision, des critères que doit satisfaire un être ou une entité pour faire l'objet d'une considération morale, etc. - problèmes de fondement qui relèvent de ce que les anglo-saxons appellent la "métaéthique ", où tous les présupposés, tous les énoncés, toutes les hypothèses constitutives de la philosophie morale sont mis à plat et examinés.

Les nouveautés éthiques qui résultent d'une interrogation menée en profondeur, et qui justifient la prétention de l'éthique environnementale à définir une éthique distincte, sont de plusieurs ordres. Afin de mieux cerner l'enjeu du différend entre les diverses 
approches des problèmes environnementaux que nous nous efforçons de présenter, nous accorderons dans ce qui suit un certain privilège au concept de "valeur intrinsèque ", qui a incontestablement exercé une influence polarisante sur une grande portion de l'éthique environnementale anglo-américaine.

L'élaboration du concept de "valeur intrinsèque » des entités du monde naturel, exigeant comme telles d'être respectées, est indissociable de la critique de fond de la tradition philosophique, religieuse, scientifique, etc., occidentale, évoquée précédemment. Pour le dire en un mot, ce qui, au sein de cette tradition, est particulièrement incriminé et objet, si l'on ose s'exprimer ainsi, de toutes les suspicions est son anthropocentrisme, c'est-à-dire l'idée selon laquelle l'être humain est la mesure de toute chose.

Lynn White Jr. est probablement le premier historien-philosophe à avoir dénoncé le caractère anthropocentrique de la culture occidentale, en liant expressément cet anthropocentrisme à la crise écologique, dans un article remarquable publié en 1967, sous le titre de «Les racines historiques de notre crise écologique » (White Jr., 1994) qui a suscité une avalanche de réponses, et est encore très discuté jusqu'aujourd'hui.

Brièvement résumée, la thèse qui y est défendue consiste à faire remonter la crise écologique à la victoire que le christianisme a remportée sur le paganisme, en laquelle Lynn White Jr. voit « la plus grande révolution psychique de notre histoire culturelle ». Dans l'Antiquité - rappelle-t-il -, chaque arbre, chaque source, chaque fleuve, chaque colline, possédait son propre genius loci, son esprit tutélaire, de sorte qu'avant de couper un arbre, de creuser une montagne ou de construire un barrage sur une rivière, il importait en tout premier lieu de se concilier l'esprit des lieux et de s'attirer ses bonnes grâces. En détruisant l'animisme païen, le christianisme a rendu possible l'exploitation de la nature dans un climat d'indifférence à l'endroit d'un environnement que les esprits et les divinités ont fui.

Plus avant, la notion de temps linéaire - que le christianisme substitue à celle de temps répétitif ou cyclique - l'idée même de Création et d'un aménagement par étapes progressives sont loin d'être indifférentes dans la mesure où elles sont à la racine de notre foi dans un progrès qui passerait par un aménagement de notre lieu de séjour, par une mainmise sur l'environnement. N'est-il pas dit expressis verbis dans la Genèse que Dieu a créé le monde pour le seul profit et pour la domination de l'Homme, auquel il appartient de « dominer sur les poissons de la mer, les oiseaux du ciel, et toutes les bestioles qui rampent sur la terre» (Genèse I, 26-29) ? Le christianisme a ainsi laissé derrière lui un monde désenchanté, un monde à former, à façonner, à dominer, un monde réduit à l'état de matière inerte, offert aux manipulations des technosciences.

31 Beaucoup de choses assurément pourraient être rétorquées à la thèse que défend Lynn White Jr., et beaucoup de choses lui ont de fait été rétorquées, mais il reste que le grand mérite de cet article est d'avoir mis, pour ainsi dire, le feu aux poudres, en invitant les différents acteurs de la communauté scientifique, considérée dans la diversité de ses champs disciplinaires, à interroger la tradition philosophique, religieuse, scientifique, etc., sous l'angle de son supposé anthropocentrisme, en ouvrant par là même de nombreux chantiers.

32 L'histoire de la philosophie ne serait-elle pas elle aussi affectée par un tel biais anthropocentriste, et ne continuerait-elle pas de l'être à son insu ? L'hypothèse n'a rien d'absurde, et il serait loisible de montrer, par exemple, que l'Homme est clairement présenté chez Aristote comme la fin dernière de la nature, en tant qu'il ne définit pas 
une forme de vie parmi les autres, mais cette forme de vie vers laquelle tendent tous les efforts de la nature, comme une sorte de chef d'œuvre de la création - moyennant quoi il prend place au sein d'une hiérarchie qui fonde en droit son empire sur l'ensemble des êtres naturels. L'Homme, parce qu'il est donné comme étant l'unique bénéficiaire de la création, est aussi bien celui qui a le droit de soumettre la nature et de l'exploiter, toute chose n'ayant par rapport à lui qu'une valeur instrumentale. Dire d'une chose qu'elle a une valeur instrumentale signifie donc exactement qu'elle n'a aucune valeur intrinsèque, aucune valeur en soi, par elle-même, mais qu'elle n'a que la valeur que lui confère l'usage que les êtres humains peuvent en avoir.

Un programme de recherche de grande envergure pourrait ainsi se fixer pour tâche de montrer dans quelle mesure cette distinction entre l'être humain qui a une valeur intrinsèque et le reste de la nature qui n'a qu'une valeur instrumentale (distinction dont nous venons de voir qu'elle procède elle-même de l'idée selon laquelle il existe un ordre naturel), traverse toute l'histoire de la philosophie, sous quelle forme elle réapparaît chez saint Thomas dans le contexte créationniste de l'échelle des êtres, comment elle est réinvestie par Kant dans la doctrine des fins en soi, etc., de sorte à faire émerger toute une tradition de philosophie morale massivement anthropocentrée, qui se révèle incapable de reconnaître une valeur autre qu'instrumentale à l'ensemble des composantes non humaines de la nature.

L'éthique environnementale se propose précisément de mettre fin à l'anthropocentrisme des valeurs qu'elle accuse d'être à l'origine des problèmes environnementaux que nous rencontrons, ce qui implique en tout premier lieu de sa part qu'elle rompe avec cette représentation de l'être humain qui consiste à le situer dans une hiérarchie de l'être qui serait un ordre de perfection. L'être humain n'est pas le rejeton choyé de la création, il est bien plutôt, pour le dire avec Aldo Leopold, « un compagnon voyageur des autres espèces dans l'odyssée de l'évolution " (Leopold, 2000, p. 145) - la théorie de la descendance avec modification et l'image de l'arbre phylogénétique venant ainsi, à point nommé, se substituer à l'idée d'une échelle des êtres dans la perspective d'une conception des rapports de l'être humain à la nature que le darwinisme a profondément renouvelée.

Dès lors qu'on en vient à se considérer soi-même comme membre d'une communauté de vie avec laquelle nous avons co-évolué, il est bien évident que le tracé des frontières de ladite communauté demande à être réexaminé. L'éthique environnementale, dont l'œuvre de Holmes Rolston III (1988) et celle de J. Baird Callicott (1999) sont probablement les plus représentatives, tient sa spécificité du fait qu'elle prend au sérieux la possibilité de concevoir les êtres vivants non humains, ainsi que les composantes abiotiques du milieu naturel, comme des patients moraux, c'est-à-dire comme des êtres susceptibles d'être présentés comme des objets de préoccupation morale pour eux-mêmes.

Par voie de conséquence, le projet d'une éthique environnementale ne peut pas être de promouvoir des comportements normatifs vis-à-vis de l'environnement non humain en fonction de la prospérité et du bien-être humain, puisque cela revient à n'attribuer de valeur à la nature qu'en fonction des êtres humains (donc une valeur instrumentale). Il n'est pas question, en éthique environnementale, de promouvoir un usage pondéré ou un usage durable des ressources naturelles pour garantir la survie de l'humanité ; il est question de revendiquer la prise en compte morale de l'environnement non humain pour lui-même, en s'opposant à la réduction des éléments qui composent 
l'environnement à de seules et uniques ressources, en vue de mettre au jour la nature comme lieu de valeurs intrinsèques dont l'existence commande un certain nombre d'obligations morales.

À terme, il semble que le système de différences que nous cherchions à faire apparaître entre les approches européennes et anglo-américaines des problèmes environnementaux ait été rendu à présent assez manifeste. Tandis que le courant de pensée écologique européen met l'accent sur l'idée de notre responsabilité dans l'usage des techniques et sur la nécessité de trouver une solution qui passerait par une recomposition du politique, l'éthique environnementale parle, elle, plus volontiers de valeurs environnementales et de respect des entités du monde naturel.

\section{Les limites réciproques des deux types d'approches: esquisse d'une théorie écologique pragmatique}

38 Conformément à ce que nous annoncions précédemment, nous n'entendons pas limiter notre propos au simple constat d'une divergence entre les méthodes de problématisation qui ont cours en matière de philosophie de l'environnement, mais nous souhaiterions exploiter le potentiel critique que porte en lui ce jeu de différences réglées, dans la mesure où il permet de révéler les insuffisances respectives de chacune des deux méthodes.

Le principe responsabilité de Hans Jonas - que nous privilégierons dans ce qui suit au titre de première grande éthique de la nature du 20e siècle, à laquelle la mort de l'auteur a conféré une sorte de clôture définitive permettant par là même à l'interprète de se prononcer en connaissance de cause - peut-il véritablement échapper au soupçon d'anthropocentrisme qu'une lecture instruite par l'éthique environnementale pourrait faire peser sur son magnum opus?

À première vue, il semble que pareil soupçon soit singulièrement injustifié concernant une œuvre qu'inspire un rejet constant de l'anthropocentrisme et qui reconnaît la communauté de destin de l'Homme et de la nature. Jonas n'écrit-il pas que l'éthique dont il forme le projet «ne peut plus s'arrêter à l'anthropocentrisme brutal qui caractérise l'éthique traditionnelle, en particulier l'éthique grecque-juive-chrétienne de l'Occident » (Jonas, 1990, p. 99), et qu'il convient d'élargir la responsabilité à la nature, en y incluant non seulement l'ensemble des vivants, mais encore le règne végétal et les ensembles écosystémiques? Ne déclare-t-il pas que "la solidarité de destin entre l'Homme et la nature [...] nous fait [...] redécouvrir la dignité autonome de la nature et nous commande de respecter son intégrité par-delà l'aspect utilitaire " (Jonas, 1990, p. 263) ? N'est-il pas l'auteur de telle page superbe, que les défenseurs les plus radicaux de la cause animale n'hésiteraient pas à contresigner, dans laquelle Jonas dénonce dans l'élevage intensif «l'avilissement ultime d'organismes doués de sens, capables de mouvement, sensibles et pleins d'énergie vitale, réduits à l'état de machine à pondre et de machines à viande, privés d'environnement, enfermés à vie, artificiellement éclairés, alimentés automatiquement » (Jonas, 1990, p. 400) ?

Quant au primat reconnu à l'Homme et assumé comme tel dans certains textes, notamment dans les articles réunis dans Le phénomène de la vie - dont on ne saurait surestimer l'importance pour l'interprétation du Principe responsabilité puisque l'éthique que développe celle-ci repose sur l'ontologie qu'explicite celle-là -, ce 
privilège résulte d'une décision méthodologique en vertu de laquelle il se pourrait bien que

l'Homme [soit] après tout la mesure de toute chose - non pas il est vrai de par la législation de sa raison, mais de par le modèle constitué par sa totalité psychophysique qui représente le maximum de complétude ontologique connue de nous, une complétude à partir de laquelle, par réduction, on devrait pouvoir déterminer la spécificité de l'être au moyen d'une soustraction ontologique progressive jusqu'au minimum de la simple matière élémentaire. (Jonas, 2001, p. 33-34)

Or les règles d'une saine méthode ne recommandent-elles pas de partir « de ce qui en dit le plus, du plus manifeste, et non du plus caché, du plus développé et non du moins développé, du plus plein et non du plus pauvre - donc du "plus élevé » qui nous soit accessible» (Jonas, 1990, p.140) - bref, de l'Homme auquel il conviendrait en conséquence de reconnaître une place à part dans l'étude des phénomènes de la vie ? Il n'y a là nul anthropocentrisme, mais au pire anthropomorphisme.

Et pourtant, dans le texte même où Jonas proclame la nécessité de mettre fin à l'anthropocentrisme brutal de la tradition occidentale, on le voit se contredire étrangement, ou du moins limiter étroitement la portée révolutionnaire d'une telle déclaration, en écrivant que :

Pour autant que l'ultime pôle de référence qui fait de l'intérêt pour la conservation de la nature un intérêt moral est le destin de l'Homme en tant qu'il dépend de l'état de la nature, l'orientation anthropocentrique de la morale classique est encore conservée ici. (Jonas, 1990, p. 32)

Si, de manière ultime, l'impératif de responsabilité entend mettre à l'abri «l'image de l'Homme ", alors nous n'avons d'obligation à l'endroit de la nature qu'en tant que le processus évolutif s'est montré capable de déboucher sur l'homo sapiens, c'est-à-dire dans la stricte mesure où notre destin coïncide avec le sien. Si l'être qui nous a produits a le droit d'exiger que ses créatures ne détruisent pas la création comme telle, c'est précisément parce qu'elle nous a produits, en sorte que l'impératif inconditionnel revient ni plus ni moins à rendre moralement contraignantes le respect des conditions générales de la survie de l'humanité. «On veut seulement dire par là » conclut à juste titre Paul Ricœur, « que l'intérêt de l'Homme coïncide avec celui du reste des vivants et celui de la nature entière en tant qu'elle est notre patrie terrestre ", ce qui n'est pas exactement la même chose que de fonder une éthique de la nature qui revendique la protection de «la plénitude de vie produite pendant le long travail créateur de la nature [...] pour son propre bien » (Ricœur, 1991, p. 215).

Il ne suffit donc pas de dire, comme le fait à l'occasion Hans Jonas, que l'Homme, parce qu'« en lui le principe de la finalité a atteint son point culminant et en même temps le point qui le menace lui-même en vertu de la liberté de s'assigner des fins et du pouvoir de les exécuter ", que l'Homme - donc - devient pour lui-même « le premier objet du devoir » (Jonas, 1990, p. 249), il faut dire qu'il est à lui-même le seul véritable objet du devoir. Hans Jonas en fait lui-même l'aveu lorsqu'il écrit qu'en ce qui a trait aux êtres qui existent indépendamment de nous, leur existence peut à la rigueur concerner notre «conscience métaphysique », mais non pas notre responsabilité, car, au fond, "pour l'essentiel, la nature prend soin d'elle-même et n'a que faire de notre approbation et de notre approbation évaluative » (Jonas, 1990, p. 249).

On dira que la rupture des équilibres naturels que provoquent les interventions humaines fait que justement la nature ne peut plus prendre soin d'elle-même, raison 
pour laquelle elle doit devenir un objet de préoccupation morale, mais aussi étonnant que cela puisse paraitre, Hans Jonas semble parfois être le premier à douter que notre pouvoir causal puisse franchir les limites que la nature pose elle-même. Ce passage proprement stupéfiant du principe responsabilité, où capitule une éthique de la nature digne de ce nom, vaut d'être longuement cité :

Demander si le monde doit être n'est pas une chose insensée, mais n'a pas grand sens, étant donné que la réponse, qu'elle soit positive ou négative, est sans conséquences : le monde existe déjà et continue à le faire ; son existence n'est pas en danger et même alors nous ne pourrions rien y faire. [...] Ce qui existe par soi et en totale indépendance de nous, son devoir-être, une fois qu'il est connu, peut sans doute importer à notre conscience métaphysique - certainement dès lors que, comme c'est le cas ici, il inclut notre existence - mais à notre responsabilité. Autre chose est la question de savoir si le monde doit être plutôt de cette façon ou de cette autre, plutôt ainsi et ainsi, car là il pourrait y avoir de l'espace pour une collaboration de notre part, et donc également pour une responsabilité et cela nous renvoie au domaine plus étroit de la causalité humaine. [...] Ce qui subsiste par sa propre force - le monde tel qu'il est - peut attendre au-delà des nécessités présentes de l'Homme et en règle générale il maintient lui-même ouverte la chance que son être-tel reçoive tôt ou tard un soutien contre le pire. (Jonas, 1990, p. 253-54)

Enfin, l'idée même selon laquelle l'Homme incarnerait une sorte de complétude ontologique - dont on ne voit pas bien, au demeurant, en quoi elle se distingue radicalement de l'ancienne théorie de l'échelle des êtres - ne laisse pas d'être extrêmement problématique, car elle semble rendre possible une discrimination non seulement entre les êtres humains et le reste de la nature, mais encore entre les êtres humains eux-mêmes en fonction de leur degré de complétude ontologique. S'il fallait appliquer la règle de soustraction ontologique dont il a été question précédemment, on voit mal comment Jonas pourrait résister à l'objection à laquelle les Anglo-Saxons ont donné le nom de l'objection ou de l'argument des cas marginaux, consistant à montrer que certains critères de considérabilité morale sont à ce point restrictifs qu'ils menacent d'exclure des groupes d'individus en les exposant par là même à un traitement inhumain. N'est-ce pas ce qui risque de se passer chez Hans Jonas dès lors que certains êtres humains (par exemple, ceux qui sont gravement handicapés) se révèlent ontologiquement inférieurs à d'autres ? L'idée de complétude ontologique estelle bien capable de fournir des principes permettant de soupeser et d'arbitrer en toute impartialité les prétentions rivales de considération morale?

48 Sur tous ces points, il faut avouer que le principe responsabilité ne livre pas de réponses claires, si bien qu'en fin de compte le soupçon d'anthropocentrisme ne disparaît jamais complètement. Sur tous ces points également, l'éthique environnementale nous paraît mieux armée, plus à même d'élaborer une éthique de la nature dont la portée écologique ne se ramène pas à la protection des conditions de la survie des êtres humains sur terre. Mais, par une sorte de retour du balancier, elle nous paraît prendre le risque de s'enfermer dans des débats proprement idéologiques et de se rendre politiquement stérile et inefficace à trop vouloir se définir elle-même par la seule référence au projet d'une éthique de la valeur intrinsèque. Comme le fait remarquer Bryan G. Norton, qui est le principal théoricien de ce que l'on appelle dans les Amériques, le pragmatisme écologique :

Énoncer de fortes propositions morales [fondées sur la considération des valeurs naturelles intrinsèques], puis entrer dans l'arène publique armé de tels principes moraux (voire moralisateurs), en se donnant pour objectif de "renforcer " par la voie de la législation un certain nombre de contraintes morales - tel n'est pas 
précisément l'état d'esprit dans lequel il convient de s'asseoir à la table des négociations dans l'espoir de trouver un compromis et de déterminer les conditions d'une action coopérative visant à assurer la protection de l'environnement. L'aplomb moral qu'insuffle la théorie de [la valeur intrinsèque objective de] Rolston est donc, à tout prendre, un handicap, car l'adoption d'une telle posture au cours des discussions des programmes de politique publique a beaucoup plus de chance de conduire à des oppositions frontales qu'à une véritable concertation et à la recherche de compromis. (Norton, 2005, p. 305)

La tâche d'évaluer des projets concurrents, d'arbitrer entre des valeurs en conflit est déjà suffisamment complexe pour qu'on ne rajoute pas une obscurité philosophique supplémentaire. De là le refus de ceux qui se rattachent au courant de pensée pragmatiste de prendre position au sein de la querelle qui oppose les partisans d'une éthique anthropocentriste à ceux d'une éthique non anthropocentriste. Ce qui compte en matière d'environnement, ce sont moins les prises de position de principe, que l'élaboration de schémas rationnels d'aide à la décision qui permettent aux différents acteurs de s'entendre sur ce qui doit être fait, en déterminant concrètement les mesures politiques qu'il convient de mettre en œuvre. En ce sens, rien n'est pire que les querelles intestines entre anthropocentristes et non anthropocentristes, humanistes et écocentristes, parce que ces querelles divisent entre eux les éthiciens de l'environnement et paralysent leur action concertée et efficace.

50 Si l'invocation exclusive du concept de valeur intrinsèque menace de se montrer contre-productive, il se peut qu'il soit bien plus utile d'élaborer un anthropocentrisme éclairé, élargi, prudentiel, qui proposerait une vision plus large des intérêts que les êtres humains peuvent avoir à préserver la nature. On ne remarque peut-être pas assez que la satisfaction des intérêts humains n'implique pas nécessairement la destruction sans retour de l'objet désiré, et qu'il importe de distinguer, sous ce rapport, entre une utilité qui se satisfait dans la consommation immédiate des biens de la nature (matières premières, produits agricoles, etc.), et une utilité qui suppose la conservation de l'objet utile en tant que la conservation est une condition de la satisfaction des intérêts humains (il en va ainsi de l'ensemble des services écologiques fournis par la nature en l'absence desquels nous n'aurions très rapidement plus aucun accès aux biens de consommation). Plus largement, la nature, loin de se réduire à n'être qu'un réservoir de matières premières ou une poubelle à ciel ouvert pour nos déchets, peut revêtir un intérêt esthétique, moral, spirituel ou scientifique au regard des êtres humains, et dans ce cas la satisfaction qu'elle procure exige que l'objet reste intact parce que la satisfaction est en quelque façon inséparable de l'objet, au point de lui être inhérente moyennant quoi il est possible de donner une signification, pour ainsi dire, pédagogique (et non plus métaphysique) au concept de valeur intrinsèque, dans la mesure où les objets de la satisfaction sont considérés comme n'étant pas indéfiniment substituables les uns aux autres.

51 Il ne s'agirait donc pas, dans la perspective d'une théorie écologique d'inspiration pragmatique, de faire l'économie du concept de valeur intrinsèque, mais de prendre acte de la pluralité essentielle des valeurs naturelles et de les situer dans une sorte de continuum, s'étirant des valeurs de la société de consommation à des valeurs esthétiques, spirituelles, intrinsèques, etc.

Dans de telles conditions, la tâche de l'environnementaliste, au moment d'entrer dans l'arène publique, devra être de définir un programme de politique environnementale qui permette la meilleure intégration harmonieuse de la gamme entière des valeurs 
naturelles. La conviction de Norton, sur ce point, est que des programmes de protection de la nature sont parfaitement justifiables du point de vue d'une conception suffisamment large de la valeur instrumentale anthropocentrique - et mieux encore: que ce dernier point de vue a une indéniable supériorité pratique, d'une part parce qu'il est le mode de justification le plus répandu chez les environnementalistes, et constitue à ce titre un espace d'interlocution immédiatement commun au sein duquel le débat pourra s'engager, et d'autre part parce qu'en neutralisant la controverse axiologique entre valeur intrinsèque et utilité humaine, il permet de laisser à la subjectivité de chacun le choix en faveur de telle ou telle option philosophique, et donc de déplacer le débat sur le terrain des modalités rationnelles d'action environnementale.

\section{BIBLIOGRAPHIE}

Anders, G. (2006). La menace nucléaire. Considérations radicales sur l'âge atomique. Paris : Le Serpent à Plumes.

Arendt, H. (1972). La crise de la culture. Paris : Gallimard.

Arendt, H. (1983). Condition de l'homme moderne. Paris : Calmann-Lévy.

Beck, U. (2001). La société du risque. Sur la voie d'une autre modernité. Paris : Flammarion.

Callicott, J. Baird (1999). Beyond the Land Ethic. More Essays in Environmental Philosophy. Albany : SUNY Press.

Ewald, F., Gollier, C. et de Sadeleer, N. (2001). Le principe de précaution. Paris : PUF.

Heidegger, M. (1980). Essais et conférences. Paris : Gallimard.

Jaspers, K. (1963). La bombe atomique et l'avenir de l'homme. Paris : Buchet-Chastel.

Jonas, H. (1990). Le Principe responsabilité. Une éthique pour la civilisation technique. Paris :

Flammarion.

Jonas, H. (1991). De la gnose au « Principe responsabilité ». Esprit, 171, 5-21.

Jonas, H. (1996). Entre le néant et l'éternité. Paris : Belin.

Jonas, H. (1997). La technique moderne comme sujet de réflexion éthique. In Neuberg, M. (dir.), La responsabilité. Questions philosophiques (p. 231-240). Paris : PUF.

Jonas, H. (1998). Pour une éthique du futur. Paris : Rivages.

Jonas, H. (2001). Le phénomène de la vie. Vers une biologie philosophique. Bruxelles : De Boeck.

Latour, B. (1991). Nous n'avons jamais été modernes. Essai d'anthropologie symétrique. Paris : La Découverte.

Latour, B. (1999). Politiques de la nature. Comment faire entrer les sciences en démocratie. Paris : La Découverte.

Leopold, A. (2000). Almanach d'un comté des sables, suivi de quelques croquis. Paris : Flammarion. 
Norton, B. (2005). Values in Nature : A Pluralistic Approach. In Cohen, A.I. et Wellman, C.H. (dir.), Contemporary Debates in Applied Ethics (p. 298-309). Oxford : Blackwell.

Ricoeur, P. (1991). La responsabilité et la fragilité de la vie. Éthique et philosophie de la vie chez Hans Jonas. Le Messager européen, 5, 203-218.

Rolston III, H. (1988). Environmental Ethics. Duties to and Values in the Natural World. Philadelphia : Temple University Press.

White Jr., L. (1994). Les racines historiques de notre crise écologique. In Goffi, J.-Y. Le philosophe et les animaux. Du statut éthique de l'animal (en appendice). Nîmes : J. Chambon.

\section{RÉSUMÉS}

L'objectif de cet essai est de proposer une lecture critique croisée de la tradition de pensée écologique européenne et de l'éthique environnementale anglo-américaine. Il s'agira de mettre au jour le manque de radicalité du type de questionnement qui prévaut en Europe, et réciproquement le risque de blocage dogmatique et d'inefficacité politique auquel s'expose l'éthique anglo-américaine de la valeur intrinsèque, en s'efforçant d'annuler les insuffisances de l'une et l'autre approche au regard d'une théorie écologique d'inspiration pragmatique.

The aim of this paper is to critically analyze environmental ethics and ecological thinking from the viewpoint of each other. A lack of radicalism within mainstream European ecological thinking is highlighted as are the risks of dogmatism and political inefficiency to which is exposed the Anglo-American intrinsic value theory, with the view of contributing to an ecological theory of pragmatic inspiration.

\section{AUTEUR}

\section{HICHAM-STÉPHANE AFEISSA}

Professeur agrégé de philosophie, Hicham-Stéphane Afeissa est rattaché à l'académie de Dijon. La phénoménologie husserlienne et l'éthique environnementale anglo-américaine constituent ses deux principaux champs de recherche. Il est l'auteur de plusieurs articles (sur Husserl, Heidegger, Kant, Fichte, les néokantiens, Norton, Rolston), et a réalisé diverses traductions (de Husserl, Heidegger, etc.). Il a récemment publié une anthologie commentée des textes fondateurs d'éthique environnementale, parue chez Vrin en 2007, et prépare actuellement un livre sur l'écologie pour le même éditeur. Membre titulaire du groupe de chercheurs en phénoménologie "Alter », il est aussi membre du comité de rédaction de diverses revues (Environmental Ethics, Alter-Revue de phénoménologie, Multitudes), et est chroniqueur pour Nonfiction.fr. depuis 2008. 